\title{
Ebola as a case study for the patent landscape of medical countermeasures for emerging infectious diseases
}

A survey of patenting activity can facilitate coordination of $R \& D$ activities to fight emerging infectious diseases.

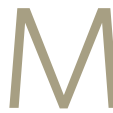

ethods to combat Ebola virus disease (EVD) have gradually improved since its discovery in 1976 (refs. ${ }^{1-3}$ ). EVD has caused over 33,000 infections and 14,000 deaths worldwide, with most cases occurring in Africa. Ebola virus (EBOV) - named for the Ebola River, located near Yambuku, Democratic Republic of the Congo (formerly Zaire)-is the cause of the disease $\mathrm{u}^{4}$. The discovery of the Reston Ebola virus in the Philippines in 1989 showed that EBOV is also present in Asia.

During the first outbreak, in 1976, improper nursing techniques such as reuse of syringes for different patients and close contact with infected blood were the main source of human-to-human transmission ${ }^{5}$. A better understanding of methods of virus transmission during the 1994 Cote d'Ivoire outbreak led to the use of personal protective equipment and single-use syringes, which helped in the control of transmission. The international public health community helped contain the disease by staffing local hospitals and providing necessary equipment and education for health care workers ${ }^{5}$.

The 2014 outbreak of EVD caused more than 28,000 cases and 11,000 deaths-more than all previous EVD outbreaks combined ${ }^{6}$. Several factors contributed to the devastating effects of this outbreak, such as the delay between identification of cases and reporting to the World Health Organization (WHO), the spread of the disease to urban areas, and the weak response of the international community ${ }^{7}$. The outbreak also highlighted lack of proper medical countermeasures (MCMs) in diagnosis of, vaccination against and treatment of the disease . $^{8}$

WHO has since included EVD on the list of priority pathogens with the potential of severe outbreaks for which few or no MCMs exist ${ }^{9}$ and has drafted an $\mathrm{R} \& \mathrm{D}$ roadmap to accelerate collaborative development of MCMs against the disease ${ }^{8}$. The vital importance of rapid isolation of infected patients based on the clinical diagnosis that could be further confirmed with the results of a readily available test
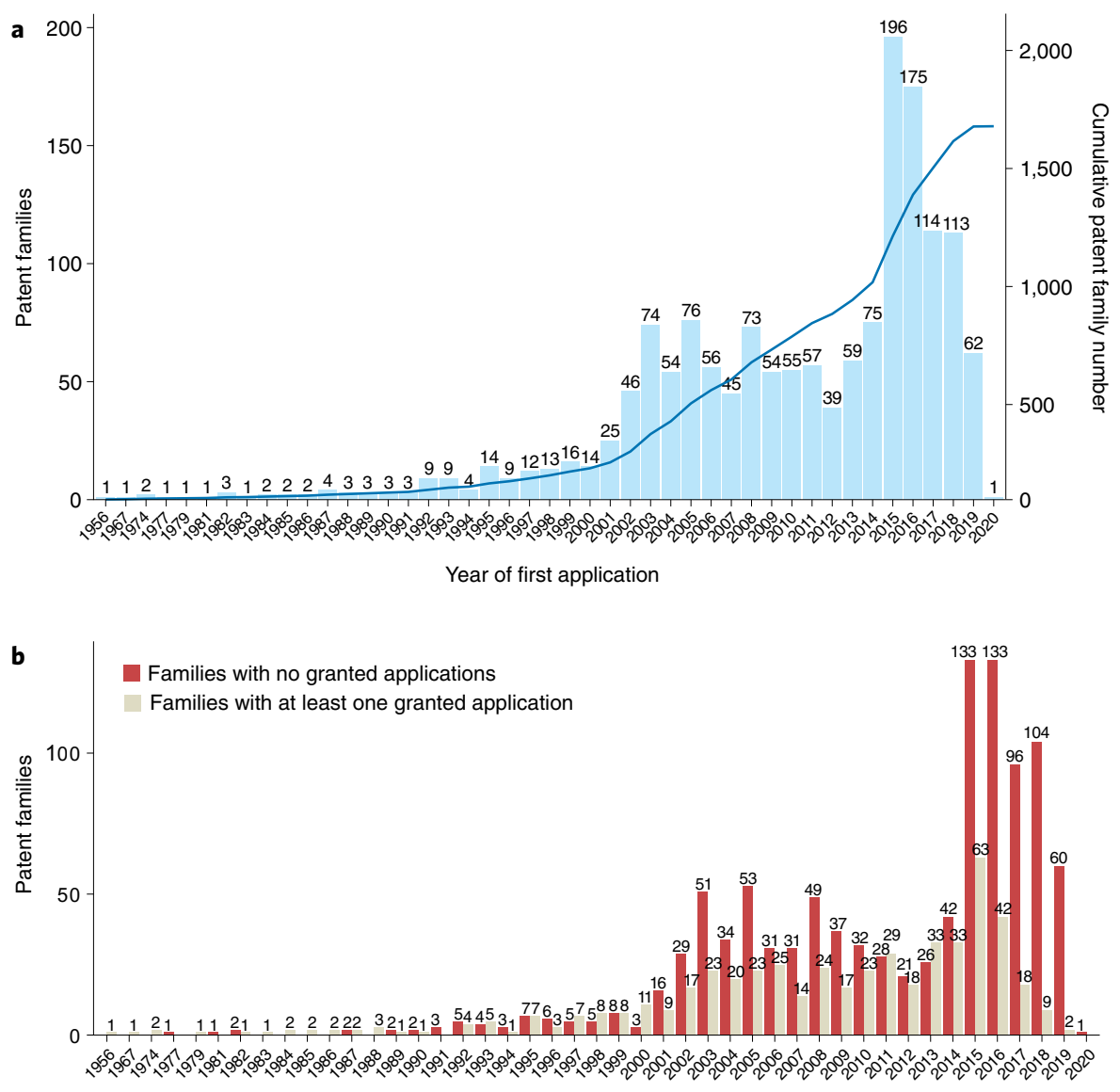

Year of first application

Fig. 1 | Analysis of patent application and granting frequencies. a, Number of patent families against application years as a bar chart and cumulative chart. There were two peaks in the number of patent applications that coincide with two EVD outbreaks. b, Families that had at least one granted patent were counted in the granted families group. The rest of the patent families were counted in no granted families group. The gap between granted and not granted patent applications widened with increase in the number of patent applications.

to control an EVD outbreak forced WHO to call for "rapid, sensitive, safe and simple EBOV diagnostic tests" as a crucial MCM in November 2014 (ref. ${ }^{10}$ ). Following this call, several tests, including various rapid diagnostic tests, became available ${ }^{4}$. PCR-based methods, which detected one or two of the EBOV genes were considered to be more specific. However, the application of rapid diagnostic tests in combination with PCR-based methods was suggested to be more effective ${ }^{11}$. Lack of data from randomized controlled trials made it difficult to draw conclusions about the efficacy of therapeutics against EVD. To address this difficulty, WHO developed an 

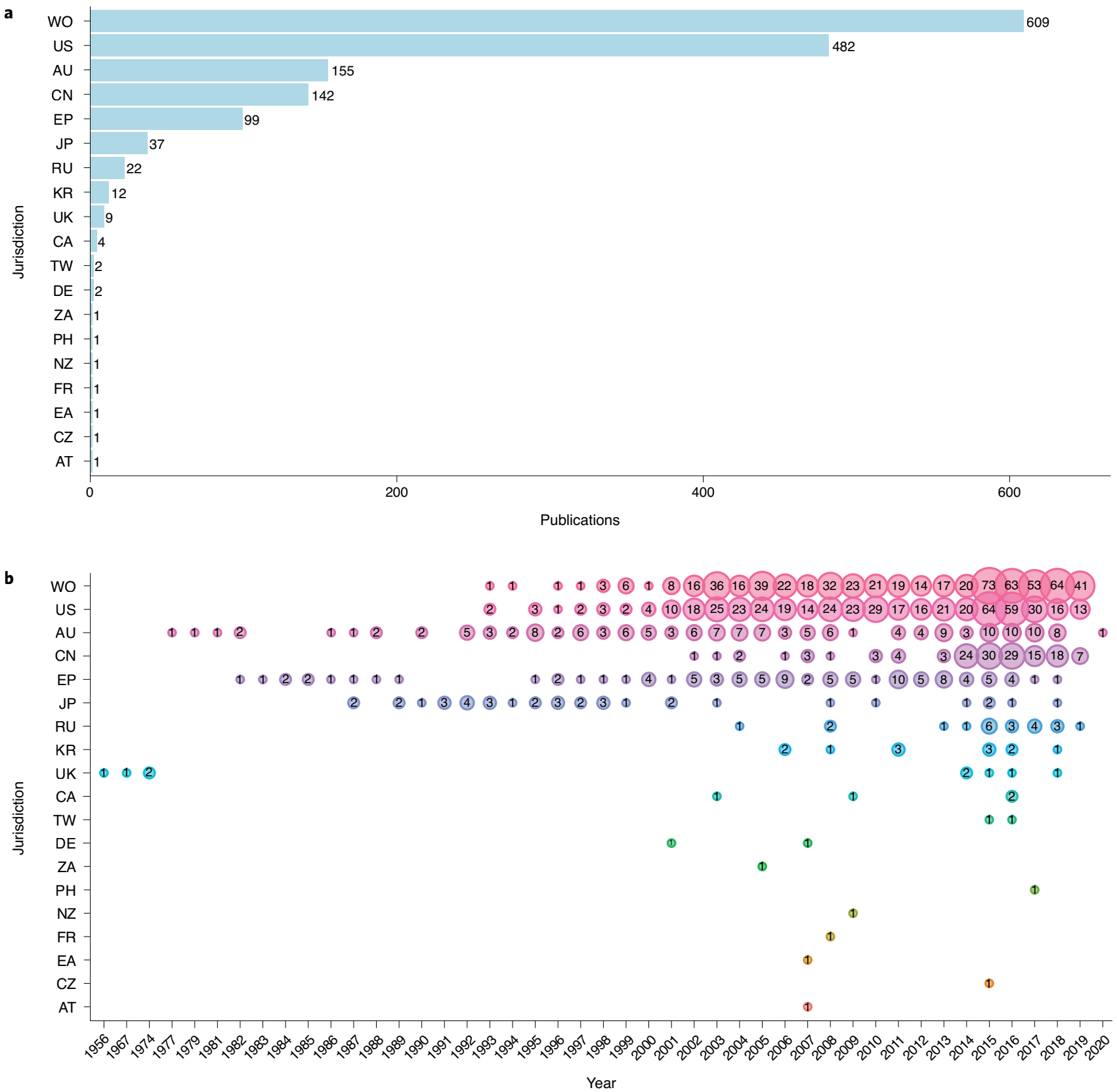

Fig. 2 | Analysis of jurisdictions. a, Offices of the first patent application are grouped and counted. The number of families that were filed through the PCT is more than in other types of applications. $\mathbf{b}$, Patent applications for each patent office plotted versus time in years. Applications in most patent offices are concentrated in the years after 2000. However, applications in some patent offices, such as AU, EP and JP, are more evenly distributed. WO, world (PCT); US, United States; AU, Australia; CN, China; EP, European Patent Office; JP, Japan; RU, Russia; KR, South Korea; UK, United Kingdom; CA, Canada; TW, Taiwan; DE, Germany; ZA, South Africa; PH, the Philippines; NZ, New Zealand; FR, France; EA, Eurasian Patent Organization; CZ, Czech Republic; AT, Austria.

ethical framework to bridge compassionate access to investigational therapeutics and clinical trials ${ }^{12}$. Following the development of this guidance, a clinical trial was initiated in the Democratic Republic of the Congo to test the monoclonal antibodies ZMapp, mAb114 and REGN-EB3, as well as the viral polymerase inhibitor remdesivir. In this trial, patients who received REGN-EB3 or mAb114 showed higher survival rates, which led to the approval of REGN-EB3, or Inmazeb (atoltivimab, maftivimab and odesivimab), as the first drug to treat $\mathrm{EVD}^{13}$.

For vaccine development, GP1,2 protein was considered the major EBOV immunogen. A recombinant vesicular stomatitis virus (VSV) pseudotyped with Ebola Zaire glycoprotein, rVSV $\Delta$ G-ZEBOV-GP, was approved by the US Food and Drug Administration (FDA) and European Medicines Agency (EMA) for the prevention of $\mathrm{EVD}^{14}$ and is being used in the Democratic Republic of the Congo according to a ring vaccination strategy. The EMA has also granted marketing authorization for a vaccine composed of a two-dose regimen of Zabdeno (Ad26. ZEBOV) and Mvabea (MVA-BN-Filo).

One of the lessons of the 2014 EVD outbreak was that collaborative development 

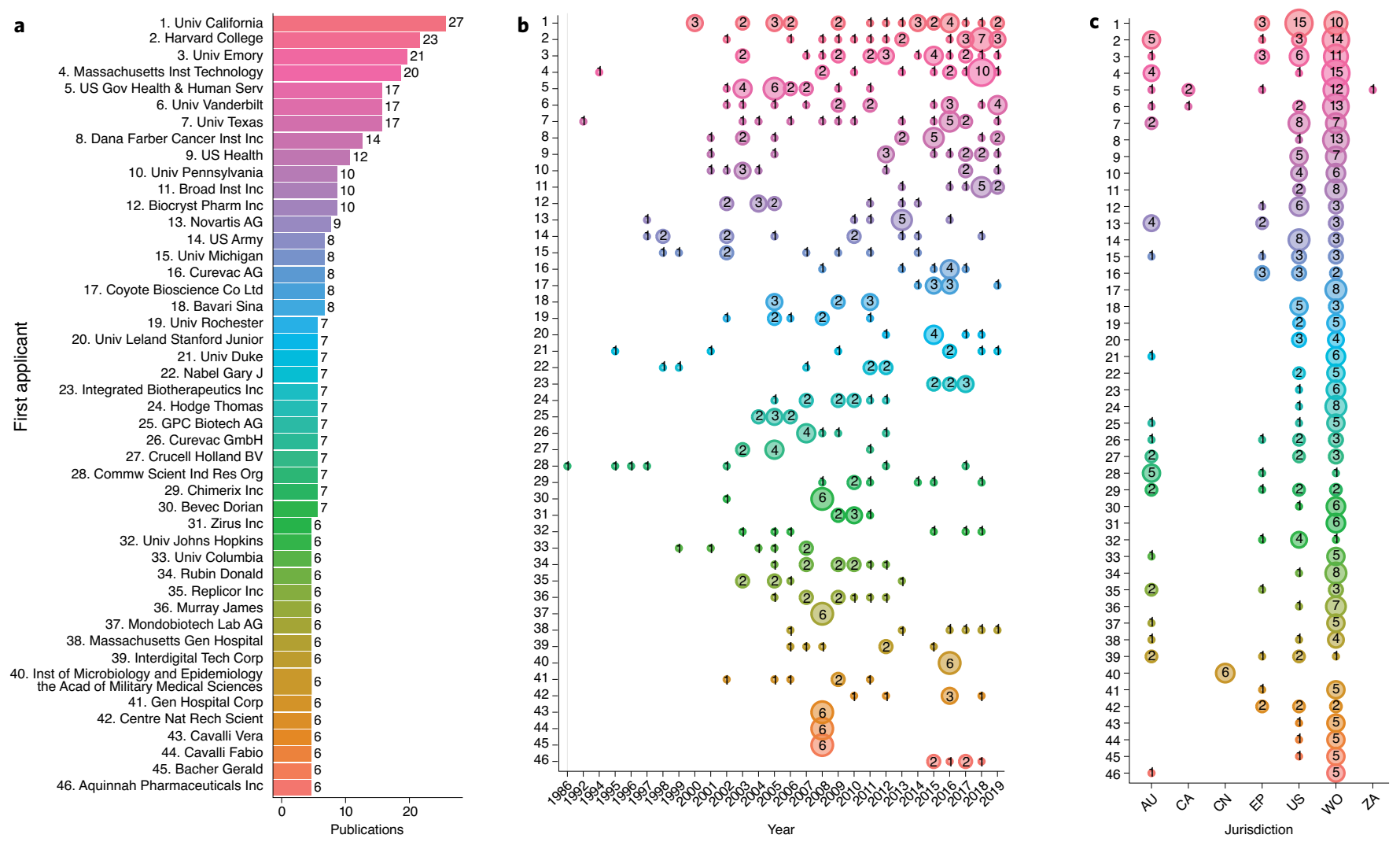

Fig. 3 Analysis of applicants with the most applications. a, Applicants in each patent family with more than five applications, plotted as a bar chart. US academic, governmental and commercial institutions are the leading applicants. $\mathbf{b}$. The number of applications in each year, graphed for the applicants in $\mathbf{a}$. Each applicant has a different patenting activity over the years. However, most patenting activity is concentrated in the years after 2000 . c, Applications by each applicant versus the office of first application. Most leading applicants prefer PCT over other patent offices for their first application.

of MCMs under the guidance of WHO can be an effective strategy to control disease outbreaks ${ }^{15}$. Major improvement on the collaborative development of MCMs could be achieved if investors, pharma companies and researchers could identify ongoing $R \& D$ activities in a technological field or against a specific disease to find collaboration opportunities and avoid parallel activities. In this regard, patent landscape reports (PLRs), the identification and analysis of issued patents and published patent applications for a particular technology or market area ${ }^{16}$, are valuable tools for data-driven decision making for various audience groups. PLRs are used by companies to assess risks imposed on their business by other patent holders, by academics and governmental agencies looking to measure the level of R\&D in a specific technological area, and by investors in company valuation ${ }^{17}$. The World Intellectual Property Office (WIPO) has published guidelines for the preparation of PLRs in an effort to make the process more standard and to help consumers of these reports better understand what to expect from them ${ }^{18}$. WIPO has also prepared PLRs in various technological areas, several of which are related to medical technologies such as vaccines ${ }^{19}$. PLRs can help to combat priority pathogens by providing global decision-makers like WHO with tools for coordinating $\mathrm{R} \& \mathrm{D}$ on various aspects of the development of MCMs against these pathogens. However, to the best of our knowledge, there has been no published PLR on patents or patent applications regarding MCMs against EVD.

Here we used freely available databases and open-source R packages to generate a PLR on MCMs developed to fight EVD. Patenting activity showed a remarkable increase after the 2001 and 2014 EVD outbreaks. The United States was dominant in the number of total applications and institutions with the most applications. Commercial institutions applied for more patents and increased their patenting activity more sharply after EVD outbreaks. Analysis of International Patent Classifications (IPCs) showed that medicinal preparations containing antigens or antibodies were most frequent among the patent families in the dataset. Finally, we used a modified network analysis tool to cluster patent families on the basis of the co-occurrence of IPCs in the same document. These clusters might be attributable to different MCMs against EVD.

\section{Results}

We used the Lens patent database (https:// www.lens.org/) to construct a database of patents related to Ebola, then used $\mathrm{R}$ packages to process and visualize different aspects of the database. We analyzed the patent application rate, granting rate, jurisdiction, applicants and classifications. We also used a modified network analysis technique to cluster patent families on the basis of the co-occurrence of IPC classifications in each patent family.

Total and granted numbers. Application rates and granting rates were obtained to assess application rate fluctuations over the years. To remove the effect of patent filing in multiple patent offices, the patent with the earliest application date was considered representative for each family. Families with more than 20 members are summarized in Supplementary Table 2. The total and the cumulative number of patent applications are shown in Fig. 1a. There are two distinct changes in patenting activity on EVD, which 
a

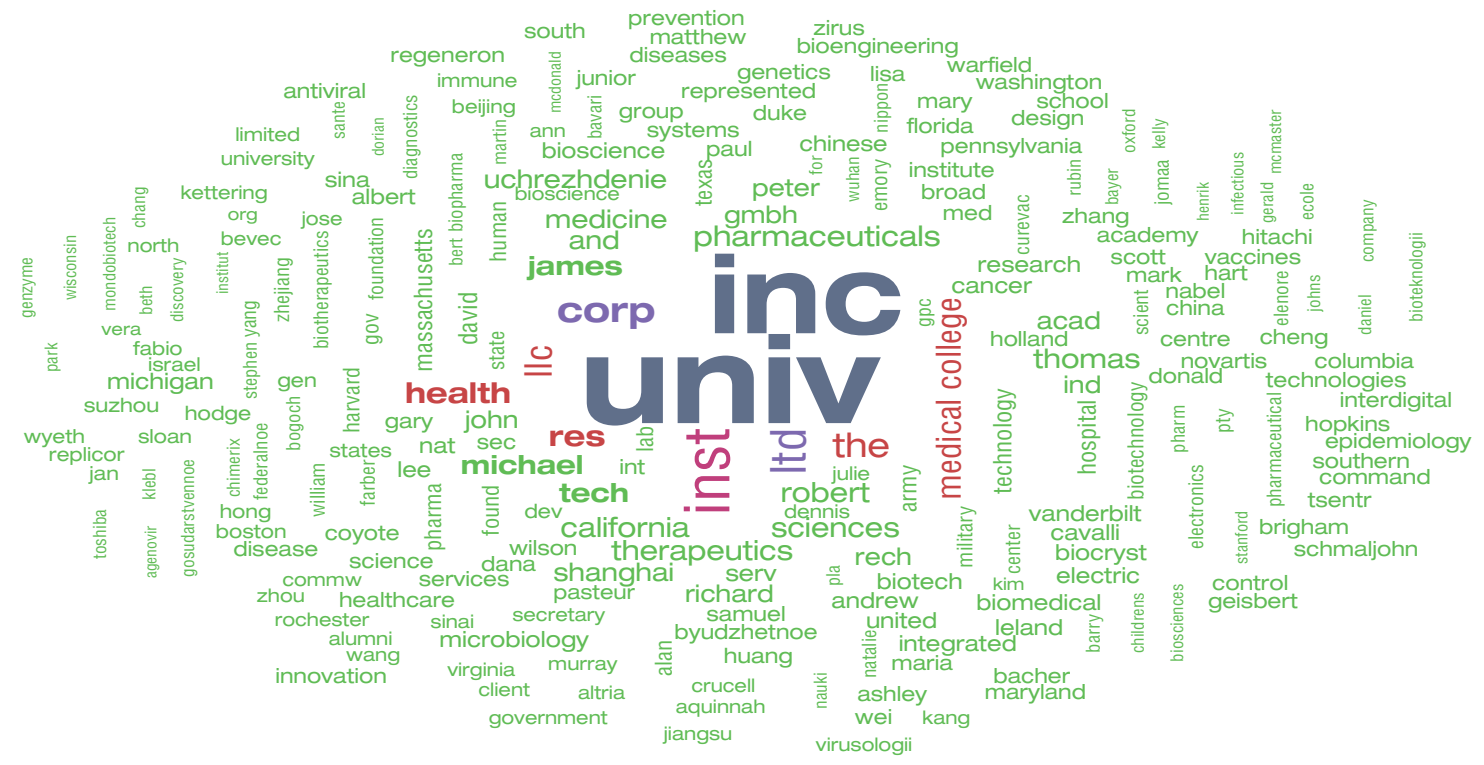

b

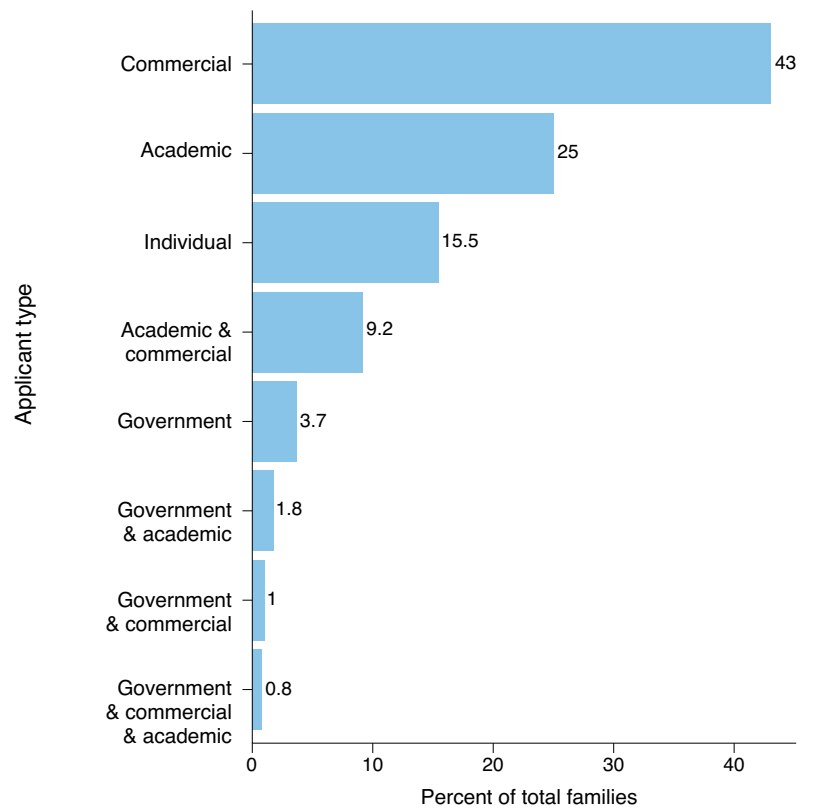

c

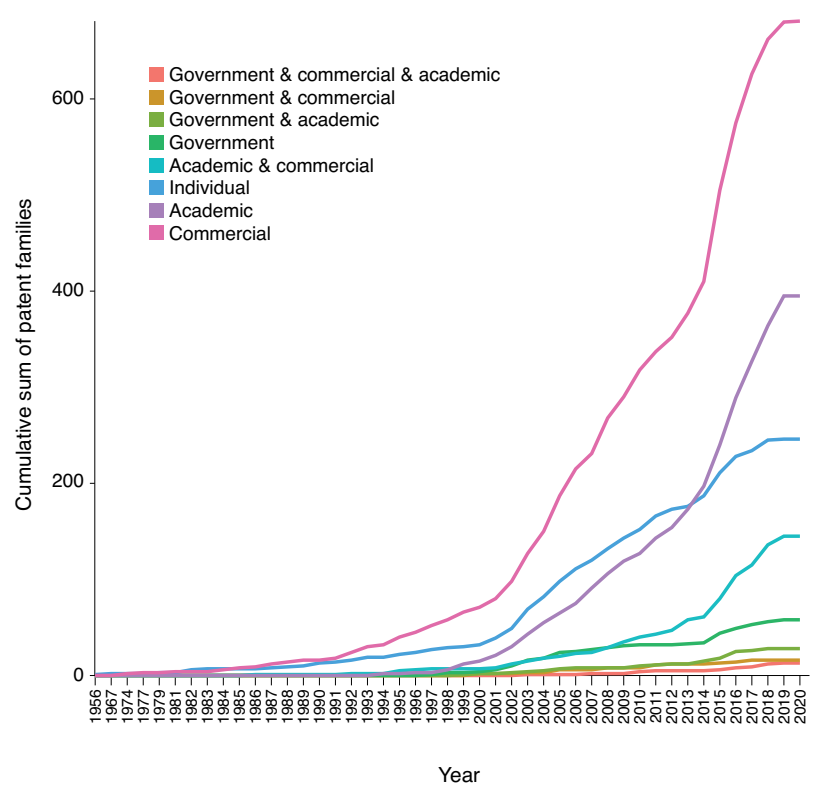

Fig. 4 | Analysis of applicant types. a, Applicants column of the patent family dataset pasted together and, after cleaning, converted into a word cloud. Commercial and academic institutions are the most abundant applicant types. b. Applications by each type of applicant, grouped on the basis of search keywords provided in Supplementary Table 1. Applications by commercial and academic institutions and collaborations therewith account for most of the applications. c, Cumulative application counts for each applicant type, plotted against year of application. Applications by commercial institutions show a greater growth rate than those by other types of applicants.

are detectable by the change in the total number of applications and in the slope of the cumulative patent applications. These increases in patenting activity coincide with two EVD outbreaks: after the 2001 outbreak on the border of Gabon and Republic of the Congo ${ }^{20}$ and after the 2014 outbreak in the Democratic Republic of the Congo ${ }^{21}$. Although the rate for granting patents follows that of the total application rate, the gap between the number of families with and without any granted applications has increased in line with the number of total applications, especially in recent years (Fig. 1b). It seems that EVD outbreaks have had a motivational effect on R\&D activities, and as a result the patent application rate has increased after them.

Jurisdictions. Since the patent with the earliest application date was chosen as the family representative, patent jurisdictions reflect the office of the first application. Applications in each jurisdiction were illustrated as a sum total and by year to determine the most favored jurisdiction for applications related to EBOV and the pattern of applications over the years.

Patent Cooperation Treaty (PCT) applications were the most abundant type of applications, followed by applications filed at the US Patent and Trademark Office (USPTO), the Australian Patent Office and the Chinese Patent Office (Fig. 2a). The pattern of patent applications was different for each patent office (Fig. 2b). Applications 
at some patent offices, such as Australia, Europe and Japan, were started earlier than those at other patent offices. PCT applications and US patent applications peaked after 2001 and 2014, similarly to the pattern of total patent applications (Fig. 1a). This pattern was not shown by applications filed in other patent offices. Patent applications that were filed before 1976 at the UK patent office were verified as search engine errors, since EBOV had not been discovered before 1976. On the basis of this information, we can conclude that PCT and USPTO are the favored offices for patent applications related to EBOV. In addition, the pattern of applications at these two offices determines the total application pattern over the years.

Applicants. Identification of applicants with the most patent applications related to EBOV can help to determine major contributors to the development of MCMs against the virus. Here, applications by each applicant were counted and applicants with more than five applications were sorted (Fig. 3a). Applications by major applicants were also plotted by year (Fig. 3b) and jurisdiction (Fig. 3c).

Three US institutions-the University of California, Harvard College and Emory University-applied for the most patent families in the dataset (Fig. 3a). The top non-US institution with the most patent applications in the field was Novartis, ranked 13th. Each institution showed a distinct application pattern over the years, and its relation to EVD outbreaks could not be established (Fig. 3b). Applicants ranked 43-45 were co-applicants for the same patent families, which resulted in the distinct pattern seen for these applicants. PCT was the favored patent application jurisdiction for major applicants (Fig. 3c). However, some applicants preferred to apply in the jurisdiction where they were located. For example, the US Army applied eight times through USPTO and three times through PCT, and the Institute of Microbiology and Epidemiology, Academy of Military Medical Science in China (ranked 40th) applied six times through the Chinese patent office. Of the most active applicants, US scientific and commercial institutions were the dominant applicants for patents related to EBOV.

Applicant type. Distinguishing the types of institutions applying for patents related to EBOV can help understand each sector's level of contribution in developing MCMs against the virus. The most common words among applicant names were identified by generating a word cloud (Fig. 4a). The ratio of applications by each type of institution-academic, commercial and governmental-was determined by matching applicants with search terms provided in Supplementary Table 1 (Fig. 4b). The cumulative rate of patent applications over the years for each type of applicant is shown in Fig. 4c.

The terms 'univ' and 'inc' were the most used terms in the word cloud, indicating that academic and commercial institutions were the most common applicants for patents related to EBOV (Fig. 4a). Commercial institutions led with 43 percent of the total applications. Moreover, commercial and academic institutions and their collaborations together accounted for 77.2 percent of total applications (Fig. 4b), in line with the results from the word cloud (Fig. 4a). In addition, the cumulative application rate by commercial institutions had a sharper slope after 2001 and 2014 than other types of applicants (Fig. 4c), which indicates a greater increase in patenting activity in response to EVD outbreaks in this sector than among other types of applicants.

Altogether, commercial institutions have had the greatest contribution to technological developments against EBOV and increased their patenting activity more than other types of applicant in response to EVD outbreaks (Fig. 4).

Technological area. To extract technological information about patents, we used IPC classifications because of their prevalent use in patent documents. We split IPCs into two parts (before and after the "/", or ipc1 and ipc2), which allowed the formation of more inclusive and general groups. The occurrence of each ipc1 was then counted in both total (Fig. 5a) and by year (Fig. 5b). The most prevalent classifications in applications by each of the major applicants are shown in Fig. 5c.

The most prevalent ipc1 among patents related to EBOV was A61K39 (Fig. 5a). Considering the most prevalent ipc2 segments of this classification (Supplementary Table 2), this classification was related to medicinal preparations containing antigens or antibodies. A61P31 (antiviral drugs) and A61K31 (medicinal preparations containing organic active ingredients) were the second and third most prevalent ipc1 segments. Distributions of the ten most prevalent ipc1 segments over the years were similar to that of total patent applications (Fig. 5b). This pattern was not uniformly followed by other ipc1 segments. The three most prevalent ipc1 segments in patent applications by the University of California (the first-ranked applicant) were A61P31 (antiviral drugs), A61K31 (medicinal preparations containing organic active ingredients) and C07F9 (compounds containing elements of groups 5 or 15 of the periodic table). Three ipc1 segments characterizing patent applications by Harvard College (the second-ranked applicant) were C12N15 (genetic engineering), C12Q1 (measuring or testing processes involving enzymes) and A61K39 (medicinal preparations containing antigens or antibodies). Patents by Novartis showed a different distribution of ipcl segments, concentrated on heterocyclic compounds (C07D401, C07D403 and C07D405).

An abstract view of Fig. 5 suggests that efforts to develop MCMs against EVD were most focused on medicinal preparations containing antigens and antibodies and also on antiviral drugs. However, the approach of each applicant was different in developing these MCMs.

Network analysis. When two IPCs are used to classify a patent, those IPCs can be assumed to be linked by that patent. These links (edges) connecting ipcl segments (nodes) are shown in Fig. 6a. A node is considered a member of a community on the basis of the number of connections it has with other nodes in the community. Community membership of the most linked nodes is summarized in Supplementary Fig. 1. Moreover, communities can be clustered on the basis of the number of nodes that they share (Fig. 6b). The nodes in each community, communities in each cluster, and a definition for each node-whether unique in the community or shared by other communities within the cluster-are provided in Supplementary Table 4.

A61P31 (anti-infectives) was the node with the most community memberships, followed by A61K31 (medicinal preparations containing organic active ingredients) and A61P35 (antineoplastic agents) (Fig. 6a and Supplementary Fig. 1), implying that anti-infectives are the common theme of all but two communities (communities number 1 and 9). These two communities formed one cluster together (Fig. $6 \mathrm{~b}$ and Supplementary Table 4), sharing the C12Q1 (measuring or testing processes involving enzymes, nucleic acids or microorganisms) node. The rest of the communities shared the A61P31 node. It can be assumed that all these communities were about anti-infectives. Communities number 2, 3 and 4 have a single unique (not shared by other communities within the cluster) node each. These nodes were C07K1 (general methods for the preparation of peptides) for number 2, A61K33 (medicinal preparations containing inorganic active ingredients) for number 3, and C07D487 (heterocyclic compounds containing nitrogen atoms as the only ring hetero atoms in the condensed 

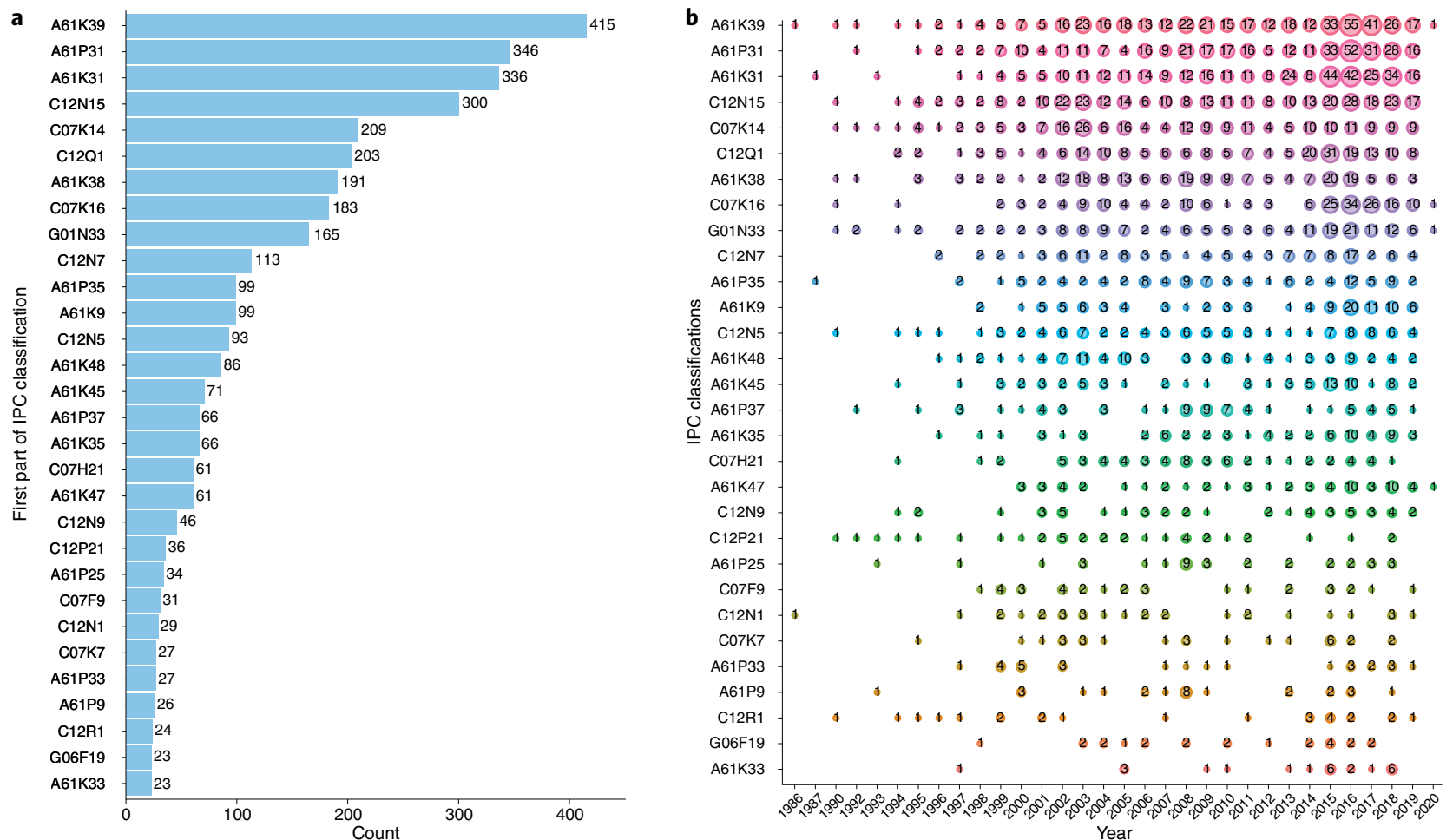

C

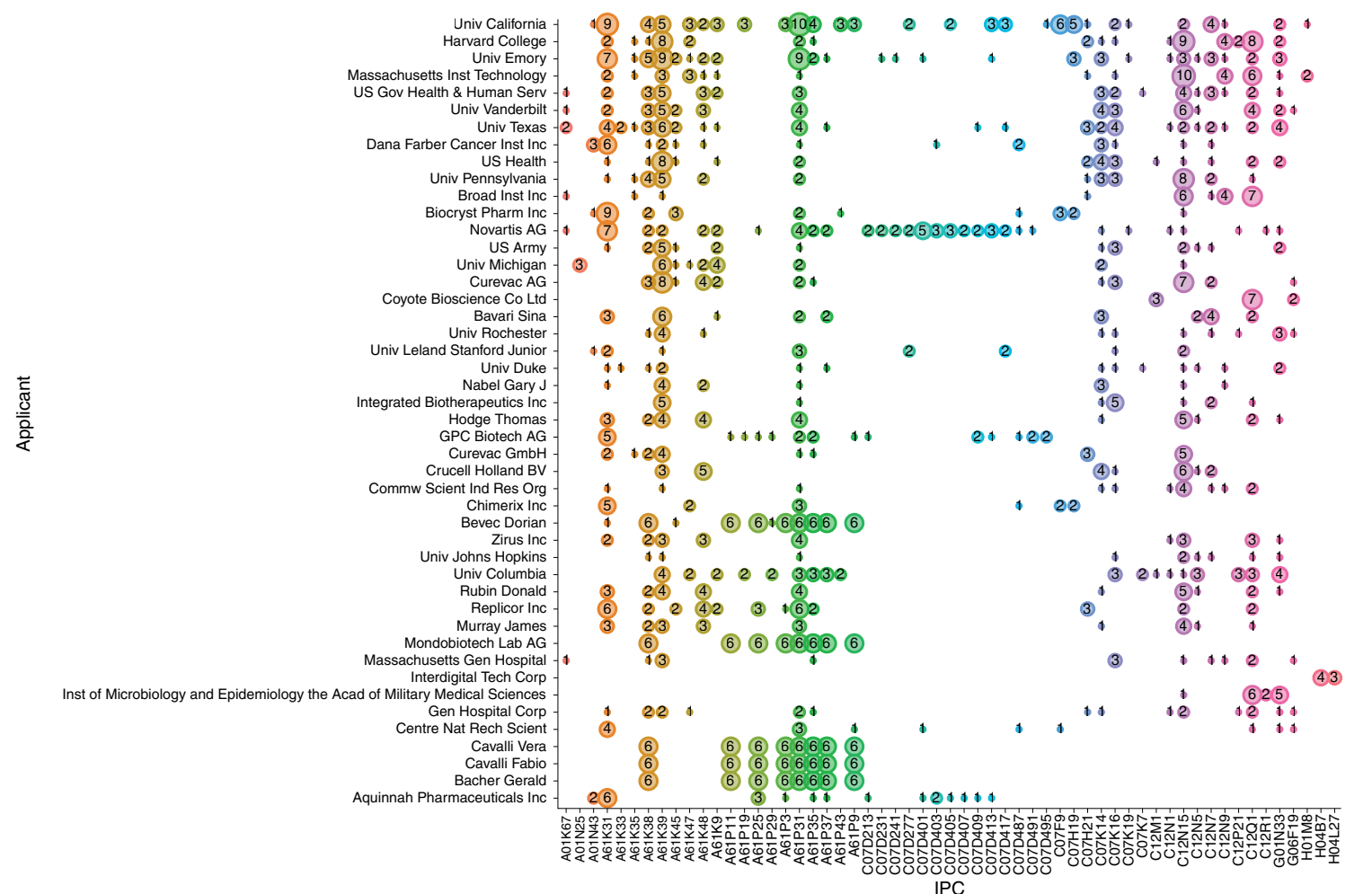

Fig. 5 | Analysis of technological areas of patent applications. a, Patent families were grouped based on their inclusion in one of the IPCs. Only the first part of the classification code (the part before "/", herein identified as ipc1) was used, to obtain more inclusive groups. The occurrence in each patent family was counted as 1, regardless of the number of times ipc1 was repeated in that family. Patent families related to antigen and antibody preparations are the most prevalent, followed by patent families related to medicinal preparations containing organic materials and patent families related to antiviral agents. Other prevalent patent families are summarized in Supplementary Table 3. b. The distribution of the most prevalent patent classifications over years. The most prevalent classification groups match the pattern of distribution of total families (Fig. 1a). However, the least prevalent classifications do not match this pattern. c, The distribution of applications by the top 30 applicants over different patent classifications. The most prevalent patent classification among the top 30 applicants is those related to medicinal preparations containing antigens or antibodies (A61K39, with 111 instances), followed by those related to genetic engineering ( $\mathrm{C} 12 \mathrm{~N} 15$, with 76 instances) and those related to medicinal preparations containing organic active ingredients (A61K31, with 71 instances). 
a

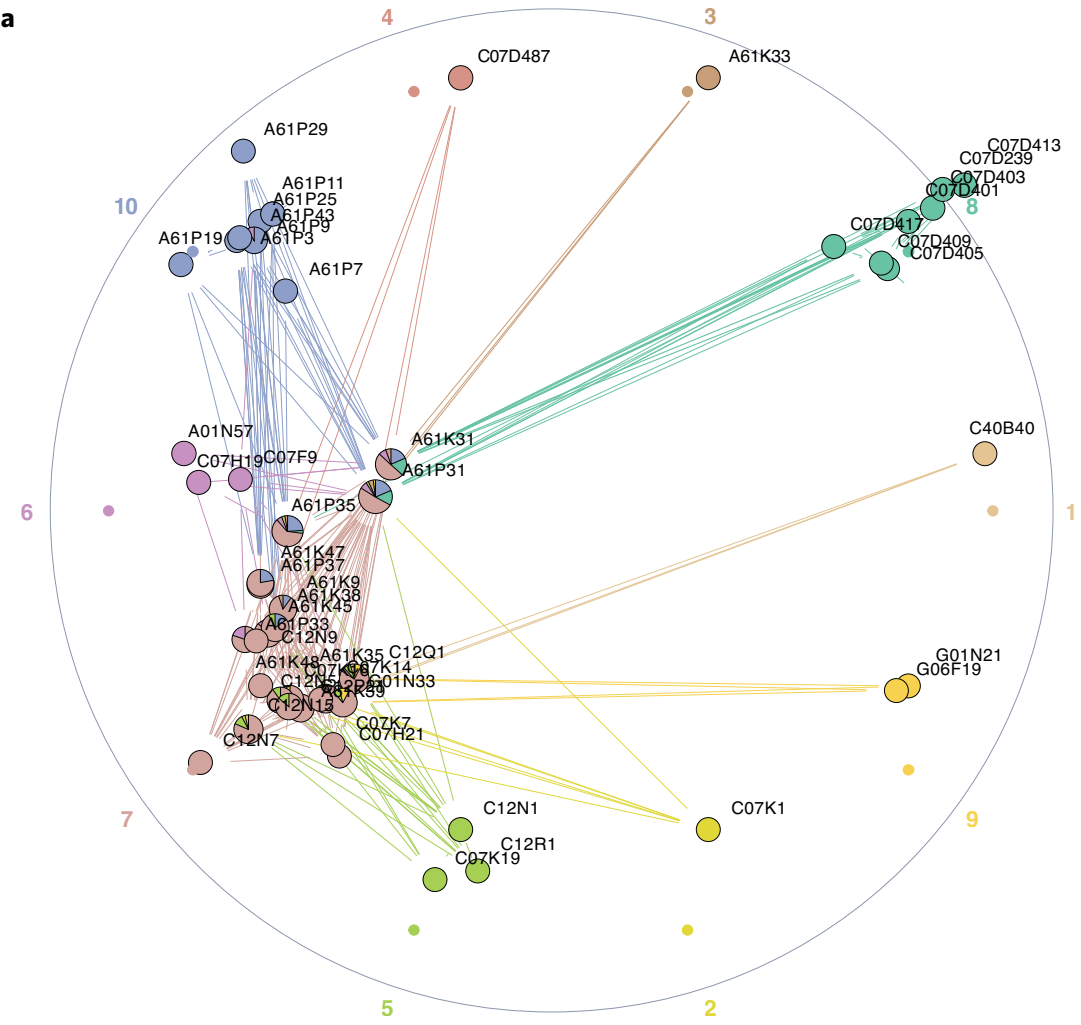

b

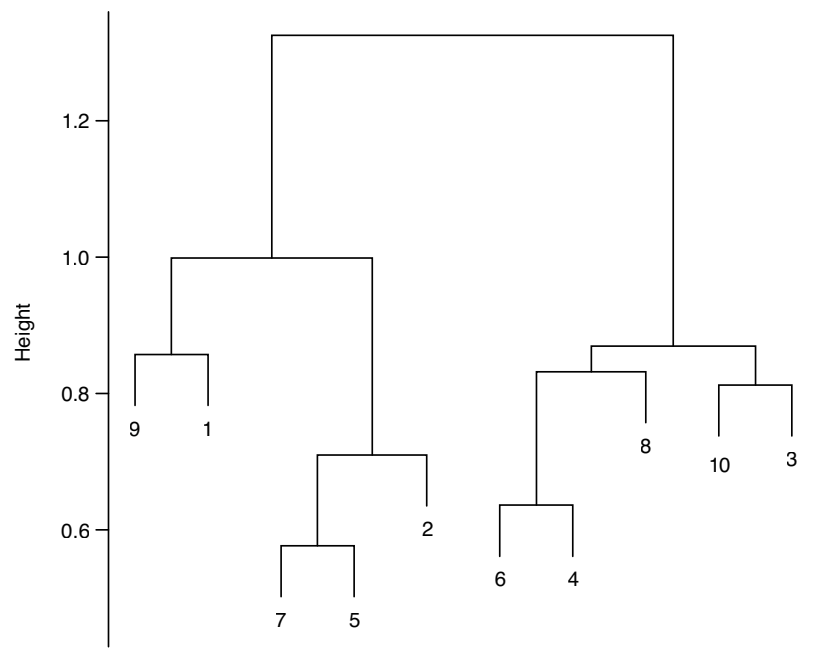

Fig. 6 | Network analysis of IPCs. a, Communities generated by the 'linkcomm' package on the basis of co-occurrence of ipc1 segments in each patent, as visualized by 'spencer.circle' layout. A61P31 (anti-infectives) and A61K31(medicinal preparations containing organic active ingredients) are two central nodes that connect all but two communities (communities 1 and 9). b. Visualization of communities from a clustered on the basis of their common nodes (ipc1 segments) using the 'getClusterRelatedness()' function from the 'linkcomm' package. There are two large clusters, which can further be divided into smaller ones.

system) for number 4 . Therefore, these communities can be defined by those nodes (Supplementary Table 4). Other communities could not be defined by a single node. However, community numbers 5,8 and 10 are populated with unique nodes that have a common technological theme: 'microorganisms', 'heterocyclic compounds' and 'drugs for various disorders', respectively. Communities number 6 and 7 did not have a technological theme in their unique nodes. On the basis of their shared nodes with the other communities in the cluster, their themes can be considered 'synthetic compounds' and 'biological materials', respectively.

Analysis of link communities showed 'detection systems' and 'anti-infectives' as two major themes in the development of MCMs against EBOV, which was in line with information gained from analysis of the prevalence of ipcl segments in patent families (Fig. 5). Anti-infectives can be divided further into biological (communities number 2, 5 and 7), synthetic (communities number 4,6 and 8) and inorganic (community number 3 ) materials. Community number 10 might be defined as inventions that claim to treat other disorders as well as EVD or inventions that were aimed at complications caused by the disease. For example, nucleoside derivatives that target viral polymerases might also be claimed as antineoplastic agents, and an invention might claim to alleviate immunological or cardiovascular complications of EVD rather than being a direct antiviral.

\section{Discussion}

We analyzed patents related to EBOV in areas of total and granted counts by year, jurisdiction, applicant and technological area. Our results show an increased number of patent applications after the 2001 and 2014 Ebola outbreaks. Applicants had a preference for PCT as their first office of application, although some major applicants preferred their national patent office for the first application. US commercial and academic institutions were the major applicants. Counting IPC classifications of patent families showed that the development of MCMs against EVD was mainly focused on medicinal preparations containing antigens and antibodies and also on antiviral drugs. These results were also confirmed with network analysis of patent classifications.

Patenting activity on a disease reflects global efforts to alleviate threats to public health imposed by that disease. The shift in patenting activity on EVD shows the mobilization of global capacities toward R\&D caused by the 2014 West African EVD outbreak. The increase in patenting activity was matched by global investment, with total investment on EVD nearly quadrupling from \$165 million in 2014 to \$631 million in 2015 (refs. ${ }^{22,23}$ ). However, patenting activity did not increase at the same rate, with only a 2.6-fold increase over the same period (Fig. 1a), possibly because the majority of funding in 2015 was dedicated to vaccine clinical trials ( $\$ 388$ million), which would not result in patent applications. 
There is also a connection between funding and patenting activity by various applicants. US public and philanthropic organizations funded 70 percent $(\$ 113$ million) of the total investment on EVD in 2014 and 50 percent ( $\$ 306$ million) in 2015 (ref. ${ }^{23}$ ). US dominance in R\&D was also evident from applications that filed at the USPTO (Fig. 2a) and the number of US institutions among the top applicants (Fig. 3a). Commercial institutions invested $20 \%$ (\$34 million) and 36\% (\$226 million) of the total investment on EVD in 2014 and 2015, respectively. Since commercial and academic institutions also received a portion of the funding by public and philanthropic institutions, one can conclude that there is also a connection between the funding spent in commercial institutions and their dominant position as applicants of EVD-related patents (Fig. 4). There is a need, however, for inclusion of the development phase in R\&D financial information to make an analysis of the relationship between investment and patenting more feasible.

Vaccines, drugs and diagnostic tests are major MCMs against EVD. However, funding in each area does not appear to be reflected in the number of patents in that area. Vaccines received the major portion of funding related to EVD in 2014 (43\%) and 2015 (61\%), followed by drugs (42\% and 16\%) and diagnostic tests (4\% in 2014 and 2015) ${ }^{23}$. It is challenging to find a relationship between investment in each MCM type and the number of patent applications related to that MCM because most patent applications are classified by more than one IPC, making attribution of the total number of patent applications for each MCM type difficult. Moreover, the reported investments include amounts that were spent in clinical trials, which are not necessary for diagnostic tests. Two different types of diagnostic tests are being used in the field: rapid viral antigen detection and PCR-based tests ${ }^{4}$. Patent applications that are classified in IPCs beginning with G01N33 (immunoassay biospecific binding assay materials) are likely to include patents related to rapid viral antigen detection tests, and patents that are classified in C12Q1 (measuring or testing processes involving enzymes, nucleic acids, or microorganisms) might include PCR-based tests (Supplementary Table 3). Whether the frequency of classification reflects the number of $R \& D$ activities on these two types of therapeutic activities is unclear because not all patent applications in G01N33 and C12Q1 classifications are related to rapid antigen detection and PCR-based tests. Furthermore, some tests might not be protected with a patent. As stated earlier, patients who received REGN-EB3 or mAb114 therapeutics in the PALM clinical trial had a better survival rate than patients who received ZMapp or remdesivir ${ }^{24}$. This study led to FDA approval of Inmazeb as the first approved drug to treat EVD. Inmazeb, mAb114 and ZMapp are based on monoclonal antibodies, and patent applications related to these and other similar drugs are classified under A61K39 and/or C07K16 (Supplementary Table 3). Remdesivir, developed by Gilead Sciences, was not shown to improve survival rate in the PALM trial but has now been approved by the FDA ${ }^{13}$ against SARS-CoV-2 as Veklury. Gilead Sciences was not among the top 40 patent applicants; however, the patent family protecting remdesivir had the most family members in our dataset (Supplementary Table 2). The number of family members is a patent value indicator because it reflects the intention of the assignee to seek protection in more jurisdictions. This patent application is classified with the following IPCs ${ }^{25}$ : C07H15/18, A61K31/00, A61K31/53, A61K31/675, A61K31/685, A61K45/06, C07D487/04, C07D519/00, C07F9/24, C07F9/6561, C07H1/00, $\mathrm{C} 07 \mathrm{H} 1 / 02, \mathrm{C} 07 \mathrm{H} 7 / 06$ and $\mathrm{C} 07 \mathrm{H} 11 / 00$. From these classifications, those beginning with C07F9 were among the most frequent classifications (Fig. 5 and Supplementary Table 3). The FDA and EMA have approved the $\mathrm{rVSV} \Delta \mathrm{G}-\mathrm{ZEBOV}-\mathrm{GP}$ vaccine to prevent EVD. The patent document protecting this invention was classified into the following $\mathrm{IPCs}^{26}$ : A61K39/12, A61K35/76, A61K39/00, C07K14/08, and C12Q1/70. Here, classifications starting with A61K39 and A61K35 were among the most frequent IPCs (Fig. 5 and Supplementary Table 3).

A better understanding of the relationship between patenting activity on each MCM and funding in that area would require further analysis, which is beyond the scope of this article. However, it is clear that patents related to each MCM cannot be counted merely on the basis of their membership in specific IPCs, and more sophisticated clustering methods similar to the one proposed here are needed.

Our results also showed that network analysis of patent classifications can be applied to cluster technologically relevant patents. This technique is also capable of revealing classifications that connect multiple clusters and therefore multiple disciplines. Network analysis has been extensively used in PLRs to reveal collaboration networks and patent citations ${ }^{27}$. In collaboration networks, the number of patents that are held in common between assignees or inventors is analyzed via network visualization tools ${ }^{28}$. Patent documents are obliged to cite prior documents that contributed to the development of the invention. Patent citation studies use network visualization tools to analyze these citation networks ${ }^{29}$. Here, we employed network analysis on a different aspect of patent documentsnamely, their classifications. Because of the diverse technologies involved in the development of MCMs against EVD, we used this technique to differentiate various technological disciplines on the basis of patent classifications and their co-occurrence in patent documents. We also used ipcl segments to allow more inclusive groups. Our results show the capability of network analysis to cluster patent documents in relevant clusters using their IPCs (Fig. 6 and Supplementary Table 4). In network analysis of the co-occurrence of patent classifications, A61K31, A61K45, C07F9 and C07D487 were members of communities number 4 and 6 , which formed a cluster. These classifications were used to classify the patent protecting remdesivir. Similarly, IPCs starting with A61K35, A61K39, C07K14 and C12Q1 were used to classify the patent protecting rVSV $\Delta$ G-ZEBOV-GP and belonged to communities number 2, 5 and 7, which also formed a cluster (Fig. 6 and Supplementary Table 4). These observations emphasize the applicability of network analysis of IPCs to the clustering of patent applications related to different MCMs. One can also use this technique in multiple steps to study a subset of the dataset. For example, any community described in Supplementary Table 4 can be chosen and analyzed again using the whole IPCs (ipc1 and ipc2 combined) to discover communities nested inside the chosen community. This could be the aim of reports that are focused on a specific technological area (for example monoclonal antibodies) that are used to fight EVD.

The vision of the Ebola/Marburg R\&D Roadmap document ${ }^{8}$ is as follows: "Robust MCMs to detect, control, and prevent outbreaks of EVD and MVD that are available, affordable, and readily deployable when needed.” Despite major advances in the areas of detection, control and prevention, many challenges remain. The introduction of rapid point-of-care tests, approval of a vaccine and conduct of several clinical trials on experimental drugs for EVD was the result of unprecedented investment in R\&D of MCMs against this disease. It is unlikely that this field can absorb the same amount of investment until the next major EVD outbreak because of the reduction of investment by commercial 
institutions, as well as redirection of funds by public and philanthropic institutions to more urgent health challenges like the ongoing COVID-19 pandemic. However, there is a need for R\&D and early screening of MCM candidates before an outbreak to provide the ability to conduct clinical trials as soon as the next possible EVD outbreak starts. As an example, the patent application for the approved vaccine against EVD (rVSV $\Delta$ G-ZEBOV-GP) had been filed in 2003 and the candidate was investigated in clinical trials during the 2014 EVD outbreak. Challenges such as scarcity of funding when there is no major active outbreak and the need to have MCM candidates prepared before the onset of such outbreaks necessitate targeted investments. Patent landscapes can be used by policymakers to identify potential solutions for those areas and redirect funding for further development of promising patent applications toward candidate MCMs ready to be investigated in clinical trials. For example, given that Inmazeb-a cocktail of three monoclonal antibodies, which is hard to provide in rural areas-is the only approved drug against EVD, the introduction of potentially more stable small-molecule drug candidates can improve accessibility and facilitate the preparation of stockpiles. Therefore, policymakers can use patent landscapes to identify major applicants working in such technological areas, look for a cluster of patents that are classified in these technological areas and focus their funding resources on these applicants or patents.
Nasir Mohajel $\mathbb{D}$ and Arash Arashkia Department of Molecular Virology, Pasteur Institute of Iran, Tehran, Iran.

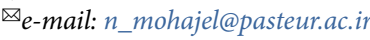

Published online: 13 July 2021

https://doi.org/10.1038/s41587-021-00970-z

References

1. Bowen, E. T. et al. Lancet 1, 571-573 (1977).

2. Johnson, K. M., Lange, J. V., Webb, P. A. \& Murphy, F. A. Lancet 309, 569-571 (1977).

3. Pattyn, S., van der Groen, G., Jacob, W., Piot, P. \& Courteille, G. Lancet 309, 573-574 (1977).

4. Jacob, S. T. et al. Nat. Rev. Dis. Primers 6, 13 (2020).

5. Amundsen, S. B. Clin. Excell. Nurse Pract. 2, 343-351 (1998).

6. Camacho, A. et al. Epidemics 9, 70-78 (2014).

7. McInnes, C. Int. Aff. 91, 1299-1316 (2015).

8. WHO. Ebola/Marburg draft R\&D roadmap. https://www.who.int/ publications/m/item/ebola-marburg-draft-r-d-roadmap (2018).

9. WHO. Prioritizing diseases for research and development in emergency contexts. https://www.who.int/activities/ prioritizing-diseases-for-research-and-development-inemergency-contexts (accessed 25 October 2020).

10. WHO. Urgently needed: rapid, sensitive, safe and simple Ebola diagnostic tests. https://web.archive.org/web/20141121152825/ https://www.who.int/mediacentre/news/ebola/18-november2014-diagnostics/en/ (2014).

11. Nouvellet, P. et al. Nature 528, S109-S116 (2015).

12. WHO. Notes for the record: consultation on Monitored Emergency Use of Unregistered and Investigational Interventions (MEURI) for Ebola virus disease (EVD). https://www.who.int/ ebola/drc-2018/notes-for-the-record-meuri-ebola.pdf (2018).

13. Office of the Commissioner. FDA approves first treatment for Ebola virus. https://www.fda.gov/news-events/pressannouncements/fda-approves-first-treatment-ebola-virus (2020).

14. US FDA. First FDA-approved vaccine for the prevention of Ebola virus disease, marking a critical milestone in public health preparedness and response. https://www.fda.gov/news-events/ press-announcements/first-fda-approved-vaccine-preventionebola-virus-disease-marking-critical-milestone-public-health (2019).

15. Henao-Restrepo, A. M., Preziosi, M. P., Wood, D., Moorthy, V. \& Kieny, M. P. Curr. Opin. Virol. 17, 138-144 (2016).

16. Weiss, K.D. \& Almeda, L.G. Competitive intelligence understanding current trends in the patent landscape for nanomaterials. 2017 IEEE 17th International Conference on
Nanotechnology (IEEE-NANO) (IEEE, 2017); https://doi. org/10.1109/nano.2017.8117271

17. Abood, A. \& Feltenberger, D. Artif. Intell. Law 26, 103-125 (2018).

18. Trippe, A. Guidelines for preparing patent landscape reports.

http://www.wipo.int/edocs/pubdocs/en/wipo_pub_946.pdf (2015).

19. WIPO. Patent landscape report on vaccines for selected infectious diseases (2012).

20. Anonymous. Wkly. Epidemiol. Rec. 78, 223-228 (2003).

21. Maganga, G. D. et al. N. Engl. J. Med. 371, 2083-2091 (2014).

22. Moran, M. et al. Neglected disease research and development: the Ebola effect. Policy Cures http://www.policycures.org/downloads/ Y8\%20GFINDER\%20full\%20report\%20web.pdf (2015).

23. Chapman, N. et al. Neglected Disease Research \& Development: A Pivotal Moment for Global Health (Health Policy Division, The George Institute for International Health, 2016).

24. Independent monitoring board recommends early termination of Ebola therapeutics trial in DRC because of favorable results with two of four candidates. https://www.nih.gov/news-events/newsreleases/independent-monitoring-board-recommends-earlytermination-ebola-therapeutics-trial-drc-because-favorableresults-two-four-candidates (2019).

25. Chun, B. K. et al. Methods for treating Filoviridae virus infections. US Patent 9,724,360 (2017).

26. Jones, S., Feldmann, H. \& Stroeher, U. Recombinant vesicular stomatitis virus vaccines for viral hemorrhagic fevers. US Patent $8,012,489$ (2011).

27. Pereira, C. G. \& Porto, G. S. in Recombinant Glycoprotein Production: Methods and Protocols (eds. Picanço-Castro, V. \& Swiech, K.) 15-34 (Springer, 2018).

28. Cantner, U. \& Graf, H. Res. Policy 35, 463-480 (2006). 29. Cho, T.-S. \& Shih, H.-Y. Scientometrics 89, 795-811 (2011).

Acknowledgements

The authors thank Sanaz Pourmohammad for assistance in preparation of the first draft.

Author contributions

N.M. searched the database, did analysis and wrote the initial draft. A.A. edited the manuscript and helped in developing the Discussion section.

Competing interests

The authors declare no competing interests.

Additional information

Supplementary information The online version contains supplementary material available at https://doi. org/10.1038/s41587-021-00970-z. 